\title{
VirTual CRIME SCEnARio ReCONSTRUCTION METHODS ASSESSMENT
}

\author{
Jiř́ Ševčík, Milan Adámek
}
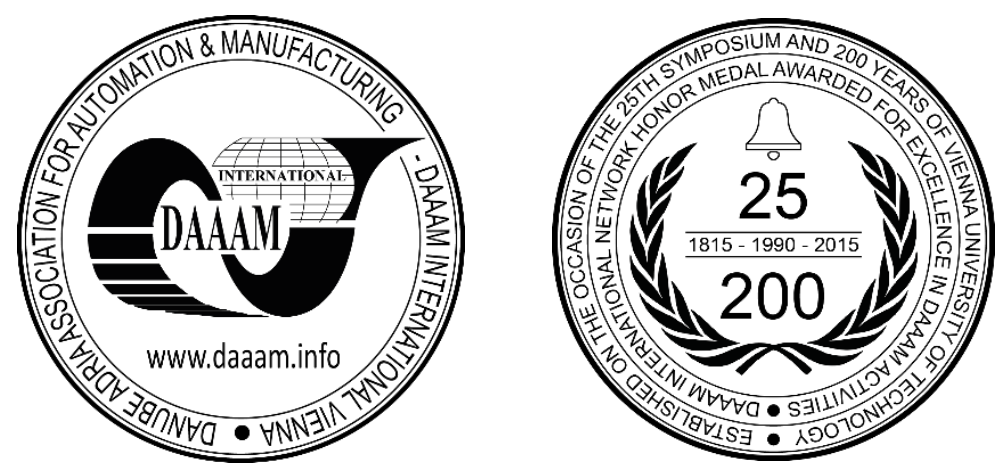

This Publication has to be referred as: Sevcik, J[iri] \& Adamek, M[ilan] (2018). Virtual Crime Scenario Reconstruction Methods Assessment, Proceedings of the 29th DAAAM International Symposium, pp.1144-1147, B. Katalinic (Ed.), Published by DAAAM International, ISBN 978-3-902734-20-4, ISSN 1726-9679, Vienna, Austria

DOI: $10.2507 / 29$ th.daaam.proceedings. 164

\begin{abstract}
Virtual environments and scenarios which are able to create fully immersive alternative visual, haptic acoustic and even olfactory experience is contained in increasing number of researches nowadays. Potential of appropriate application could be very high. Very specific fusion of sciences is described in this paper. Particularly, immersive virtual reality and technology of polygraph connection considerations are analysed, moreover the framework of this synergy is proposed. Furthermore, suitable methods of virtual crime scenario fast reconstruction are reviewed and possible next research steps are exposed.
\end{abstract}

Keywords: immersive virtual environment; crime scenario; polygraph

\section{Introduction}

Recent progress in virtualization of the real environment, including the scenarios that can possibly take place in it, draws the attention of both the professional and the scientific community. The application of virtual reality (VR) aspires to the post of reformer in terms of the natural connection of man and computer technology. VR could be defined as an advanced user interface that includes real-time simulation of real environment where the user is interacting through several sensory channels: sight, hearing, touch, smell and taste. The more human body sensors are involved, the more authentic is the VR experience. Applied research, where virtualization is utilized to simulate reality in particular form. Solution of appropriate methods and techniques specific for individual application of VR is currently addressed in several researches. Significant benefit in diverse range of human activities is expected via suitable utilization of this progressive technology. The most expanding areas include health care, where VR is used to treat mental illness, where patient could adopt variety of traumatic situations. Other medical kind application is proposed for immersive simulation of surgical procedures, where undoubtedly helps to accelerate education and reaching specialist practice. Nonetheless, availability of an incomparably higher computational and graphical performance compared to the 1990s and early 21st century gives rise to a new space for the development of specific applications applicable not only from the point of view of education by simulation but also from the point of view of inducing certain changes in perception and the state of mind.

When the attraction of human internal feelings, perception and natural intuitive manifestation is main intention the immersive virtual environment (VE) has to be constructed or reconstructed. 
The level of influence of technical precision of real environment virtual interpretation has to be evaluated only by statistic conclusion of experimentally obtained results. Moreover, there are other perspectives which are important to include, when the immersive ability of VR is assessed. Particular research topics are aimed to induction of feel of immersion, where the process of transition into the VR is described [1]. Virtual environment design process could be realized by two major approaches. First one is the proposal and construction of brand new unique VE. There are variety of methods of VE creation, nonetheless the one most utilized is raw 3D modelling often done within Unity 3D, Unreal Engine or other 3D modelling tools [14]. The second is aimed to reconstruction of real environment into the virtual one, where all relevant characteristics are interpreted in appropriate, purpose related way.

Contemporary research in VR is rather empirical, based on experimental applications of human and machine interaction. Virtual crime scene reconstruction represents relatively fast evolving area which is closely connected to general VE reconstruction. Progressive approach of 3D crime scene reconstruction throughout interactive investigation tool by using photogrammetry technique was presented by Bostanci [2]. Reality skins introduce their tool for creating immersive and tactile virtual environments, where combination of color and depth camera was utilized [3]. Another research paper aimed to utilisation of spherical photography and virtual tour for crime scene representation. It is obvious, that the visual factor is playing the most important role within the crime scene reconstruction, and that the static environment is sufficient for purposes of 3D digital evidence representation.

Nevertheless, by term crime scenario which is described on this research paper is meant VE which is dynamic, where particular action taking a place and where defined act is depicted. The principal objective of this VE is to evoke physiological and cogitation changes on persons who are immersed within it.

\section{Virtual Crime Scenario for Detection of Lie}

The issue of crime scene reconstruction has been discussed in previous chapter but important is to define the difference between scene and scenario. The scene is static 3D interpretation which could be observed from variety of different angles, but it remain stable, without any movement of objects within and without any particular process or action is depicted. On the other hand the scenario is representation of particular process within the virtual environment which based on real area reconstruction.

The intention of research is utilisation of connection of immersive ability of virtual scenarios and methods of scanning human physiological and psychological characteristics. To reach these characteristics variety of methods could be used, nonetheless a polygraph, well known as lie detector was considered for the beginning phase. Other brain scanning methods like an fMRI (Functional Magnetic Resonance Imaging), EEG (Electro-Encephalography) are considered to the future research phases.

\subsection{Lie Detector}

A polygraph, popularly referred to as a lie detector, is a device or procedure that measures and records several physiological indicators such as blood pressure, pulse, respiration, and skin conductivity while a person is asked and answers a series of questions [4]. The intention of this research is to transfer questions for immersive virtual crime scenario. Lie detector is also utilized within legislative of my homeland Czech Republic as a supporting evidence within judgement process.

\subsection{Evaluation Criteria Specification}

Main purpose of virtual crime scenario is to create immersive virtual environment as an accurate reflection of real space. Usually the action of crime has been an action depicted in. The question is, which method will suit the best for purposes of application described. There are several VE reconstruction methods utilised within virtual scenario reconstruction:

- Photogrammetry,

- 3D laser scanning techniques (LiDAR),

- RGB- D mapping,

- 3D game engines (Unreal 4, Unity 3D).

Firstly, specification of relevant evaluation characteristics has to be accomplished to get possibility to consider suitability of particular methods. One of the most important is speed of reconstruction. Another is level of visual authenticity, which directly influence the level of virtual scenario immersion. The design severity on both SW/HW and human powers has to be maintained and represents third significant evaluation factor. Otherwise, scalability and versatility parameters are describing the ability of particular reconstruction methods to be applicable in the most possible situations and define last evaluation factor. For summarisation, particular virtual crime scenario reconstruction method will be assessed through following evaluation criteria: 
- Speed of 3D reconstruction,

- Level of visual authenticity,

- Design severity,

- Scalability and versatility.

Generally, each of reconstruction methods which are presented in the paper has its advantages and disadvantages and the most appropriate seems to be interconnection of two or more of them or its sequential application within scenario generation phases. Introduction of particular methods and their assessment according to specified evaluation criteria is provided in next section.

\section{3D Reconstruction Methods Assessment}

Realistic model of three dimensional space including all necessary object and characteristics, which will be designed in fastest possible way, will be versatile and possible to work in environment of police department is critical task for the available methods of contemporary research. Firstly, particular methods description is provided.

\subsection{Photogrammetry}

Photogrammetry uses methods from many disciplines, including optics and projective geometry. Digital image capturing and photogrammetric processing includes several well defined stages, which allow to generate 2D or 3D digital models of the object as an end product [5].The essence of an image is a projection from a 3D scene onto a 2D plane, during which process the depth is lost. The 3D point corresponding to a specific image point is constrained to be on the line of sight. From a single image, it is impossible to determine which point on this line corresponds to the image point. If two images are available, then the position of a 3D point can be found as the intersection of the two projection rays. This process is referred to as triangulation. The key for this process is the relations between multiple views which convey the information that corresponding sets of points must contain some structure and that this structure is related to the poses and the calibration of the camera [6].

Depth determination serves as the most challenging part in the whole process, as it calculates the 3D component missing from any given image - depth. The correspondence problem, finding matches between two images so the position of the matched elements can then be triangulated in 3D space is the key issue here. Once you have the multiple depth maps you have to combine them to create a final mesh by calculating depth and projecting out of the camera - registration. Camera calibration will be used to identify where the many meshes created by depth maps can be combined together to develop a larger one, providing more than one view for observation. By the stage of Material Application you have a complete 3D mesh, which may be the final goal, but usually you will want to apply the colour from the original photographs to the mesh. This can range from projecting the images onto the mesh randomly, through approaches of combining the textures for super resolution and finally to segmenting the mesh by material, such as specular and diffuse properties [6].

\subsection{D laser scanning}

The purpose of a 3D scanner is usually to create a 3D model. This 3D model consists of a point cloud of geometric samples on the surface of the subject. These points can then be used to extrapolate the shape of the subject (a process called reconstruction). If colour information is collected at each point, then the colours on the surface of the subject can also be determined [13].

3D scanners share several traits with cameras. Like most cameras, they have a cone-like field of view, and like cameras, they can only collect information about surfaces that are not obscured. While a camera collects colour information about surfaces within its field of view, a 3D scanner collects distance information about surfaces within its field of view. Output produced by a 3D scanner describes the distance to a surface at each point in the picture. This allows the three dimensional position of each point in the picture to be identified.

For most situations, a single scan will not produce a complete model of the subject. Multiple scans, even hundreds, from many different directions are usually required to obtain information about all sides of the subject. These scans have to be brought into a common reference system, a process that is usually called alignment or registration, and then merged to create a complete 3D model. This whole process, going from the single range map to the whole model, is usually known as the 3D scanning pipeline [7].

\subsection{RGB-D Mapping}

RGB-D cameras are sensing systems that capture RGB images along with perpixel depth information. RGB-D cameras rely on either active stereo $[8,9]$ or time-of-flight sensing $[10,11]$ to generate depth estimates at a large number of pixels. While sensor systems with these capabilities have been custom-built for years, only now are they being packaged in form factors that make them attractive for research outside specialized computer vision groups. In fact, the key drivers for the most recent RGB-D camera systems are computer gaming and home entertainment applications [8]. 


\subsection{D Game Engines}

The Unreal 4 and Unity 3D are the most popular 3D engines nowadays. Virtual environment could be created manually through these engines. Creating of virtual world including all necessary models could be realized in relatively simple way by drag and drop graphical user interface. The results of engines are widely utilized in computer games, but also in several VR applications. The problem in relation to the $3 \mathrm{D}$ scenario reconstruction lies in high time consumption and there are also difficulties in creating an authentic replication of real site. Nonetheless, there are some possibilities to use these engines in combination with previously described methods [12].

\section{Conclusion}

The novel approach of VR and polygraph application was proposed within the paper. Nonetheless the research is in the beginning phase. Software and hardware accessory, which is necessary for further evaluation were chosen and should be available soon. That is one of reasons, why this study is rather theoretical. Deeper practical evaluation of methods described will be then possible. Nevertheless, background information required for further research were summarised and the evaluation criteria of existing methods were proposed. According to this study the next research phase could start by executing empirical based steps.

\section{References}

[1] Sproll, Daniel, Jacob Freiberg, Timofey Grechkin a Bernhard E. Riecke (2013). Poster: Paving the way into virtual reality - A transition in five stages. In: 2013 IEEE Symposium on 3D User Interfaces (3DUI)[online]. IEEE, 2013, s. 175-176 [cit. 2018-10-19]. DOI: 10.1109/3DUI.2013.6550235. ISBN 978-1-4673-6098-2. Dostupné z: http://ieeexplore.ieee.org/document/6550235/

[2] Bostanci, Erkan. (2015). 3D Reconstruction of Crime Scenes and Design Considerations for an Interactive Investigation Tool.

[3] Shapira, Lior \& Freedman, Daniel (2016). Reality Skins: Creating Immersive and Tactile Virtual Environments. 115-124. 10.1109/ISMAR.2016.23.

[4] J P Rosenfeld (1995). "Alternative Views of Bashore and Rapp's (1993) alternatives to traditional polygraphy: a critique". Psychological Bulletin. 117: 159-66. doi:10.1037/0033-2909.117.1.159

[5] Sužiedelytė-Visockienė J, Bagdžiūnaitè R, Malys N, Maliene V (2015). "Close-Rage Photogrametry Enables Documentation of Environment-Induced Deformation of Architectural Heritage"

[6] A. Laurentini (February 1994). "The visual hull concept for silhouette-based image understanding". IEEE Trans. Pattern Analysis and Machine Intelligence. pp. 150-162.

[7] Fausto Bernardini, Holly E. Rushmeier (2002). "The 3D Model Acquisition Pipeline" (pdf). Comput. Graph. Forum. 21 (2): 149-172. doi:10.1111/1467-8659.00574

[8] K. Konolige (2010) Projected texture stereo. In Proc. of the IEEE International Conference of Robotics \& Automation (ICRA),

[9] PrimeSense. http://www.primesense.com/.

[10] Canesta. http://www.canesta.com/.

[11] Mesa Imaging. http://www.mesa-imaging.ch/

[12] Kharin, K[onstantin]; Kuvshinov, S[ergey]; Pryanichnikov, V[alentin] E[.] \& Anufrieva, E[leonora] (2017). Implementation of Augmented and Virtual Reality Technologies for an Educational and Research Situation Center, Proceedings of the 28th DAAAM International Symposium, pp.0361-0366, B. Katalinic (Ed.), Published by DAAAM International, ISBN 978-3-902734-11-2, ISSN 1726-9679, Vienna, Austria

[13] Martirosov, S[ergo] \& Kopecek, P[avel] (2017). Virtual Reality and its Influence on Training and Education Literature Review, Proceedings of the 28th DAAAM International Symposium, pp.0708- 0717, B. Katalinic (Ed.), Published by DAAAM International, ISBN 978-3-902734-11-2, ISSN 1726-9679, Vienna, Austria

[14] Andreev, V[iktor]; Karbanov, V[iacheslav]; Kharin, K[onstantin]; Kuvshinov, S[ergey]; Poduraev, Y[ury] \& Pryanichnikov, V[alentin] (2016). Training Situation Center Based on Three-Dimensional Virtual Studio for Distributed Mobile Robotics Laboratory, Proceedings of the 26th DAAAM International Symposium, pp.04830487, B. Katalinic (Ed.), Published by DAAAM International, ISBN 978-3-902734-07-5, ISSN 1726-9679, Vienna, Austria 